\title{
International Journal of Health Services Research and Policy
}

www.dergipark.org.tr/ijhsrp

IJHSRP

e-ISSN: 2602-3482

Research Article

\section{DOES THE FREQUENCY OF ACUTE CHOLECYSTITIS DECREASE DURING THE RAMADAN MONTH?}

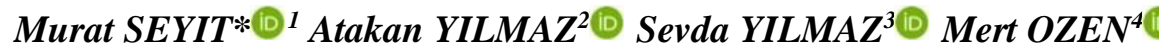 \\ ${ }^{1}$ Pamukkale University, Medical Faculty, Department of Emergency Medicine, 20070, Denizli, Turkey \\ ${ }^{2}$ Pamukkale University, Medical Faculty, Department of Emergency Medicine, 20070, Denizli, Turkey \\ ${ }^{3}$ Pamukkale University, Medical Faculty, Department of General Surgery, 20070, Denizli, Turkey \\ ${ }^{4}$ Pamukkale University, Medical Faculty, Department of Emergency Medicine, 20070, Denizli, Turkey \\ *Corresponding author; muratseyit@yahoo.com
}

\begin{abstract}
The pathophysiological mechanism of acute cholecystitis (AC), which is essentially the inflammation of the gallbladder, is blockage of the cystic duct, and its most common cause is gallstones. On the other hand, fasting held in Ramadan is worship practiced by Muslims within the period from dawn to sunset by avoiding any eating and drinking act. Within this framework, our study ultimately intends to reveal the variation of $A C$ in two different time periods. We retrospectively compared the demographic characteristics, admittance times, biochemical parameters of the subjects diagnosed with $A C$ and hospitalized in the general surgery between 2012 and 2018. We also evaluated diagnosis classification, length of hospitalization, presence of intensive care stay, presence of operation during hospitalization, and the underlying comorbid diseases of the subjects. 225 patients were hospitalized in the general surgery service after being diagnosed with AC in the ED over the course of six years, yet the total number of subjects recruited for the study is 46 . Whereas 10 patients (21.7\%) were admitted and hospitalized during the Ramadan month, 36 (78.3\%) patients in the other group were hospitalized one month before and after Ramadan. 16 (34.8\%) of the hospitalized patients underwent emergency surgery, while $30(65.2 \%)$ patients were operated on electively. The comparison of the two cohorts in our study revealed no significant difference in relation to the demographic characteristics, laboratory findings, imaging methods, underlying comorbid diseases, and the length of hospital stay. Nevertheless, clinical investigations seem to have revealed a difference between groups.
\end{abstract}

Keywords: Acute cholecystitis, General surgery, Abdominal pain

Received: June 10, 2021

Accepted: October 4, 2021

\section{Introduction}

The pathophysiological mechanism of acute cholecystitis (AC), which is essentially the inflammation of the gallbladder, is the obstruction of the cystic duct, and its most common cause is gallstones. The diagnosis of this disease should be confirmed by radiological imaging methods in patients with clinically suspected AC. Besides, cholecystitis is a condition that is best treated with surgery but can also be treated conservatively, if necessary.

Under the Tokyo Guidelines 2018, the diagnostic criteria of AC are made up of three components (Table 1) (1) presence of local signs of inflammation (e.g. Murphy's sign or pain or tenderness in the right upper quadrant); (2) signs of systemic inflammation (e.g. fever, increased C-reactive protein [CRP], increased white blood cell [elevated WBC]), and (3) imaging findings [1]. The suspected 
diagnosis is made through the co-existence of one of local inflammation and systemic inflammation findings, while the definitive diagnosis is made with the presence of imaging findings.

Table 1 Tokyo Guidelines 2018, the diagnostic criteria of Acute Cholecystitis

TG18/TG13 Diagnostic Criteria For Acute Cholecystitis

A. Local signs of inflammation etc.

(1) Murphy's sign, (2) RUQ mass/pain/tenderness

B. Systemic signs of inflammation etc.

(1) Fever, (2) elevated CRP, (3) elevated WBC count

C. Imaging findings

Imaging findings characteristic of acute cholecystitis

Suspected diagnosis: one item in $\mathrm{A}+$ one item in $\mathrm{B}$

Definite diagnosis: one item in A + one item in B + C

RUQ: Right Upper Quadrant; CRP: C-Reactive Protein; WBC: White Blood Cell

The $8^{\text {th }}, 9^{\text {th }}$, and $10^{\text {th }}$ months are sequenced as Shaban, Ramadan, and Shawwal, respectively, according to the lunar calendar (Hijri), in which one month lasts 29-30 days and one year consists of 354 days. Therefore, the month of Ramadan falls 10-11 days earlier than the previous year, judging by the Gregorian calendar. Fasting held in Ramadan is worship practised by Muslims within the period from sunrise to sunset, which generally covers 11-19 hours, by avoiding any eating and drinking act. Smoking, taking oral intra-muscular or intravenous drugs, eating and drinking, and performing sexual acts are strictly prohibited during the worship of fasting [2]. Prior to the fasting period, some preparations are made through the consumption of food and water, and the fasting is ended on that day with the evening adhan (sunset) by eating dinner. Here, the purpose is to curb the cravings of the flesh. Under the teachings of Islam, anyone who reaches puberty, maintains their mental health, does not have an underlying chronic disease and does not travel long distances is supposed to fulfill their fasting ritual.

A review of the literature suggests that the amount of research into the association between the month of Ramadan and cholecystitis is rather limited indeed, and the existing studies on this phenomenon have observed only seasonal variations. With this in mind, the present study set out to unravel the association between acute cholecystitis and the month of Ramadan.

\section{Materials and Methods}

The approval for this study was granted from the non-interventional ethics committee of Pamukkale University with the decision number 60116787-020 / 34888 dated April 17, 2018, and numbered 2018/08. Following a retrospective review of the archive, this study eventually included over 18-year-old patients diagnosed with AC and hospitalized in the general surgery service between January 2012 and December 2018 in the Emergency Department (ED) of a hospital. This retrospective study intended to reveal the difference between the patients diagnosed with AC during the Ramadan and nonRamadan period. Considering that there is no seasonal variation because the month of Ramadan is celebrated 10-11 days earlier than the previous year according to the Gregorian calendar [3], we compared the subjects in the month of Ramadan as one group with those in the month of Shaban (one month before Ramadan) and the month of Shawwal (one month after Ramadan) as the other group.

\subsection{Data collection}

The demographic characteristics, admission times, white blood count, alanine aminotransferase (ALT), aspartate aminotransferase (AST), C-Reactive Protein (CRP), direct bilirubin, indirect bilirubin, and cholesterol values of the subjects diagnosed with $\mathrm{AC}$ and hospitalized in the general surgery service in the ED of a hospital between 2012 and 2018 were retrospectively compared using the ICD (International Classification of Diseases) code. The diagnosis classification, the length of hospital stay, the status of hospitalization in the intensive care unit (ICU), the status of undergoing an operation during 
hospitalization, and the underlying comorbid diseases of the subjects were also investigated within the scope of the present study.

\subsection{Statistical analysis}

Performed by using IBM SPSS Statistics 22 documentation program, the statistical analyses are primarily based on descriptive statistics, such as mean scores, standard deviation, minimum, maximum, and percentage values. The patients were divided into two cohorts and compared as Group 1, consisting of the subjects admitted in the Ramadan month, and as Group 2, including the subjects admitted in the pre-Ramadan (Shaban) and post-Ramadan (Shawwal) months. Shapiro Wilk test were used for determination of normal distribution. For independent groups comparisons, we used Independent samples $t$ test when parametric test assumptions were provided, Mann Whitney $U$ test were used when parametric test assumptions were not provided. Difference between categorical variables were analyzed with Fisher Exact test. Statistical significance was determined as $\mathrm{p}<0,05$.

\section{Results}

Although the number of patients diagnosed with AC in the ED and hospitalized in the general surgery service between 2012 and 2018 amounted to 225, the total number of patients included in the study was ultimately 46. The number of within-Ramadan (Group 1) and non-Ramadan (Group 2) subjects were $10(21.7 \%)$ and $36(78.3 \%)$ respectively.

18 of the subjects were male (39.1\%), and 28 subjects were female $(60.9 \%)$. Besides, the mean age of the subjects was 59.5 ( \pm 16.45$)$ (min. 24 and max. 88).

As far as the diagnosis classification is concerned, 7 subjects were hospitalized after being diagnosed with calculous cholecystitis and 3 subjects with acalculous cholecystitis in Group 1, while 24 subjects were diagnosed with calculus cholecystitis and 12 subjects with calculus cholecystitis were hospitalized in Group 2.

While $43(93.5 \%)$ of the hospitalized patients were followed up in the general surgery service, 3 (6.5\%) patients admitted during the non-Ramadan months were observed to require intensive care.

Whereas $16(34.8 \%)$ of the hospitalized patients underwent an emergency operation, $30(65.2 \%)$ patients were operated on electively. In addition, 1 patient subjected to emergency surgery was hospitalized during the Ramadan month, while the remaining 15 patients were hospitalized during the non-Ramadan months.

While diagnosing patients with AC, ultrasonography imaging was performed in the ED for 30 patients $(65.2 \%)$ (7 in Group 1), abdominal tomography imaging for $22(47.8 \%)$ patients (2 in Group 1), MRI for 14 (30.4\%) patients (2 in Group 1) and other imaging for 8 (17.4\%) patients (2 in Group 1).

Given the underlying comorbid diseases of the subjects, 11 (23.9\%) of them were suffering from hypertension ( 2 in Group 1), 3 of them (6.5\%) were afflicted with diabetes mellitus (no diagnosis of diabetes in Group 1), and 3 of them (6.5\%) had COPD (no diagnosis of COPD in Group 1). In addition, ischemic heart disease was identified in $2(4.3 \%)$ patients (no diagnosis of ischemic heart disease in Group 1), while 10 (21.7\%) patients were afflicted with different chronic diseases (no diagnosis of different chronic diseases in Group 1).

As for the laboratory outcomes of the subjects, the mean score for white blood count was established to be $11669( \pm 5452)(13295[ \pm 3435]$ in Group 1). On the other hand, the mean AST value was found as $108.91( \pm 181.98)(93.8[ \pm 115.05]$ in Group 1$)$, the mean ALT value as $106.34( \pm 163.53)$ (124 [ \pm 176.17$]$ in Group 1), and the mean CRP value as 10.53 ( \pm 11.55$)(8.36[ \pm 10.95]$ in Group 1). Furthermore, the mean direct bilirubin level was calculated as $0.67( \pm 16.85)(1.08[ \pm 1.43]$ in Group 1), while that of indirect bilirubin turned out to be $0.76( \pm 1.04)(1.12[ \pm 1.77]$ in Group 1). Finally, the 
mean cholesterol level was identified as $141.14 \mathrm{mg} / \mathrm{dl}( \pm 31.33)$ (136 [ \pm 8.84$]$ in Group 1), yet it was realized that cholesterol levels were not measured in every subject included in the study.

The average length of hospital stay of the subjects was established as 8.11 days $( \pm 5.14)$ (min. 2 days and max. 23 days) in Group 2, whereas this average was found as 5.6 days ( \pm 1.84 ) (min. 3 days and max. 9 days) in Group 1.

Among the three subjects followed up in the intensive care unit were an 82-year-old male patient, an 83-year-old female patient, and a 78-year-old male patient. The length of hospital stay for these three subjects was 19, 10, and 15 days, respectively. All of them were admitted to the ED during the nonRamadan months and underwent an emergency operation. While the first patient was diagnosed with calculous cholecystitis, the diagnosis of the other two patients was established as acalculous cholecystitis.

As the group comparisons reveal clearly, no significant difference was identified between the demographic features, laboratory findings, imaging methods, comorbid diseases, and the length of hospital stay in our study (Table 2).

Table 2. Demographic features, laboratory findings, imaging methods, comorbid diseases, and the length of hospital stay

\begin{tabular}{|c|c|c|c|c|c|}
\hline & \multicolumn{2}{|c|}{ Ramadan } & \multicolumn{2}{|c|}{ Non-Ramadan } & \multirow[b]{2}{*}{ p (Test) } \\
\hline & $\mathrm{X} \pm \mathrm{SD}$ & Med (Min-Max) & $\mathrm{X} \pm \mathrm{SD}$ & Med (Min - Max) & \\
\hline Age & $59.5 \pm 16.45$ & $53.5(40-86)$ & $59.47 \pm 16.72$ & $58(24-88)$ & $>0.05(\mathrm{t}=0.005)$ \\
\hline Hospital stay/day & $5.6 \pm 1.84$ & $5.5(3-9)$ & $8.11 \pm 5.63$ & $6(2-23)$ & $>0.05(\mathrm{z}=-1.092)$ \\
\hline WBC & $13295 \pm 3435.67$ & $12900(8770-20220)$ & $11217.66 \pm 5849.39$ & $10640(6.87-27000)$ & $>0.05(t=1.068)$ \\
\hline $\begin{array}{l}\text { AST (aspartate } \\
\text { aminotransferase) }\end{array}$ & $93.8 \pm 115.05$ & $44.5(16-359)$ & $113.11 \pm 197.72$ & $41.5(13-1078)$ & $>0.05(\mathrm{z}=-0.04)$ \\
\hline $\begin{array}{l}\text { ALT (alanine } \\
\text { aminotransferase) }\end{array}$ & $124 \pm 176.17$ & $33(8-544)$ & $101.44 \pm 162.14$ & $25.5(3-764)$ & $>0.05(\mathrm{z}=-0.799)$ \\
\hline Direct bilirubin & $1.08 \pm 1.43$ & $0.35(0.08-3.82)$ & $0.55 \pm 0.78$ & $0,24(0.05-3.92)$ & $>0.05(\mathrm{z}=-1.066)$ \\
\hline Indirect bilirubin & $1.12 \pm 1.77$ & $0,35(0.04-5.67)$ & $0.66 \pm 0.73$ & $0.54(0.01-4.36)$ & $>0.05(\mathrm{z}=-0.386)$ \\
\hline \multirow[t]{3}{*}{ CRP } & $8.36 \pm 10.95$ & $4.43(0.3-31.78)$ & $11.03 \pm 11.79$ & $7.77(0.08-39.14)$ & $>0.05(\mathrm{z}=-0.499)$ \\
\hline & & \multicolumn{2}{|c|}{ Group } & & \\
\hline & & Ramadan & Non-Ramadan & Total & \\
\hline \multirow{2}{*}{ Sex } & Male & $6(60 \%)$ & $12(33.33 \%)$ & $18(39,13 \%)$ & $>0.05(\delta=2.337)$ \\
\hline & Female & $4(40 \%)$ & $24(66.67 \%)$ & $28(60,87 \%)$ & \\
\hline \multirow{2}{*}{ Ultrasound } & Yes & $7(70 \%)$ & $23(63.89 \%)$ & $30(65,22 \%)$ & $>0.05(\delta=0.129)$ \\
\hline & No & $3(30 \%)$ & $13(36.11 \%)$ & $16(34,78 \%)$ & \\
\hline \multirow{2}{*}{$\begin{array}{l}\text { Computerized } \\
\text { tomography }\end{array}$} & Yes & $2(20 \%)$ & $20(55.56 \%)$ & $22(47,83 \%)$ & $>0.05(\delta=3,965)$ \\
\hline & No & $8(80 \%)$ & $16(44.44 \%)$ & $24(52,17 \%)$ & \\
\hline \multirow{2}{*}{$\begin{array}{l}\text { Magnetic } \\
\text { resonance }\end{array}$} & Yes & $2(20 \%)$ & $12(33.33 \%)$ & $14(30,43 \%)$ & $>0.05(\delta=0.667)$ \\
\hline & No & $8(80 \%)$ & $24(66.67 \%)$ & $32(69,57 \%)$ & \\
\hline \multirow{2}{*}{ Other imaging } & Yes & $2(20 \%)$ & $6(16.67 \%)$ & $8(17,39 \%)$ & $>0.05(\delta=0.061)$ \\
\hline & No & $8(80 \%)$ & $30(83.33 \%)$ & $38(82,61 \%)$ & \\
\hline \multirow{2}{*}{ Operation } & Emergency & $1(10 \%)$ & $15(41.67 \%)$ & $16(34,78 \%)$ & $>0.05(\delta=3.460)$ \\
\hline & Elective & $9(90 \%)$ & $21(58.33 \%)$ & $30(65,22 \%)$ & \\
\hline \multirow{2}{*}{ Diagnosis } & Calculosis & $7(70 \%)$ & $24(66.67 \%)$ & $31(67,39 \%)$ & $>0.05(\delta=0.040)$ \\
\hline & Acalculosis & $3(30 \%)$ & $12(33.33 \%)$ & $15(32,61 \%)$ & \\
\hline \multirow{2}{*}{ Hypertension } & Yes & $2(20 \%)$ & $9(25 \%)$ & $11(23,91 \%)$ & $>0.05(\delta=0.106)$ \\
\hline & No & $8(80 \%)$ & $27(75 \%)$ & $35(76,09 \%)$ & \\
\hline \multirow{2}{*}{ Diabetes } & Yes & $0(0 \%)$ & $3(8.33 \%)$ & $3(6,52 \%)$ & $>0.05(\delta=0.891)$ \\
\hline & No & $10(100 \%)$ & $33(91.67 \%)$ & $43(93,48 \%)$ & \\
\hline
\end{tabular}


Table 2. Continued

\begin{tabular}{llcccc}
\hline & \multicolumn{2}{c}{ Group } & \multicolumn{2}{c}{ Total } & \\
\hline Chronic obstructive & Yes & $0(0 \%)$ & $3(8.33 \%)$ & $3(6.52 \%)$ & $>0.05(\delta=0.891)$ \\
pulmonary disease & No & $10(100)$ & $33(91.67 \%)$ & $43(93.48 \%)$ & \\
Other chronic & Yes & $0(0 \%)$ & $10(27.78 \%)$ & $10(21.74 \%)$ & $>0.05(\delta=3.549)$ \\
disease & No & $10(10 \% 0)$ & $26(72.22 \%)$ & $36(78.26 \%)$ & \\
Coronary artery & Yes & $0(0 \%)$ & $2(5.56 \%)$ & $2(4.35 \%)$ & $>0.05(\delta=0.561)$ \\
disease & No & $10(100 \%)$ & $34(94.44 \%)$ & $44(95.65 \%)$ & $>0.05(\delta=0.891)$ \\
Intensive care unit & Yes & $0(0 \%)$ & $3(8.33 \%)$ & $3(6.52 \%)$ & \\
\hline
\end{tabular}

WBC: White Blood Cell; CRP: C-Reactive Protein; t: Independent Samples t test; z: Mann Whitney U test; $\delta$ : Fisher Exact test

\section{Discussion}

In their study on the association of AC with Ramadan fasting and its seasonal variation, Hosseini et al. [3] reported that the frequency of AC remained higher during the summertime than other seasons, noting no significant difference in its frequency during Ramadan in comparison to Shaban and Shavval months.

In Valerie's systematic review, AC was detected in $20 \%$ of the cases admitted to the hospital due to biliary tract disease. AC was reported to be three times more common in female subjects than their male counterparts until the age of 50, yet this ratio was observed to decrease to one and a half times after the age of 50. As a side note, it was highlighted that approximately $95 \%$ of the subjects with AC also had gallstones [4]. In our study, the ratio of women to men afflicted with AC was identified as one and a half times, when the age of 50 was accepted as the cut-off age as in Valerie's review study. However, our proportion of acute calculous cholecystitis among our patients diagnosed with AC was found as $67.4 \%$, which is lower than the proportion reported in Valerie's review. In this regard, the fact that fasting state and dehydration are cited as the primary triggers of acalculous cholecystitis [5] may account for the higher rate of this disease in Group 1.

Early cholecystectomy to be administered within seven days after the onset of symptoms is the ideal treatment for AC [4]. In a clinical review by Indar et al [6], 20\% of the AC sufferers were reported to have required emergency surgery. According to the researchers, this situation might have resulted from the deterioration of the patient's condition, the presence of general signs of peritonitis, and gangrened or perforated gallbladder. When it comes to our study, emergency surgery was performed in $34.8 \%$ of the study population, which seems to be a higher rate in comparison to that reported by Indar et al.'s review. More specifically, $1(10 \%)$ patient in Group 1 and 15 patients (41.67\%) in Group 2 were subjected to emergency operation in our study $(\mathrm{p}=0.13)$. Although the $\mathrm{p}$-value for this finding is 0.13 and it is statistically non-significant, and although the smaller number of patients operated in Group 1 is not statistically significant, a clinical difference is nevertheless observable numerically. We tend to attribute the reason for the smaller number of emergency operations during Ramadan to postponing the surgery until the post-holiday period, devoting enough time to holiday preparations, and not staying in the hospital during the holiday. We also assume that the general surgeon following up with the patients might have preferred to postpone the surgery during Ramadan and that the patient may have also wanted to postpone it due to holiday preparations, to meet his relatives during the festive and not to stay at the hospital during the holiday.

In their study on the impact of seasons and fasting practiced during Ramadan upon the emergence of AC, Hosseini et al [3] hold that gallstone-induced AC might manifest different seasonal characteristics, but that this situation may also arise from other environmental factors. It is further suggested that abstaining from water and other liquid substances throughout Ramadan fasting may not 
trigger the onset of the disease. In the years included in this study, the month of Ramadan coincided with the summer period between May 15 and August 18, thus we compared the subjects admitted during the month of Ramadan as one group and the subjects admitted one month before and after the Ramadan as the other group in order to eliminate the seasonal variation. Nevertheless, no significant difference was noted between the two groups ( $\mathrm{p}<0.05$ ). Our subjects were evaluated based on a seven-year period, almost all of which coincided with the summer months. We are of the opinion that more extensive studies should be undertaken by making more detailed comparisons covering all months, rather than focusing on a specific period of time.

Ultrasound can be exploited to evaluate the presence of ductal dilatation or fluid collection [7]. In their meta-analysis, Abboud et al [8] report that abdominal ultrasound could reach $42 \%$ sensitivity and $96 \%$ specificity for enlarged bile duct while showing $38 \%$ sensitivity and $100 \%$ specificity for all bile duct stones. These results demonstrate that abdominal ultrasound tends to achieve high specificity but suffers insufficient sensitivity. On the other hand, $65.2 \%$ of the patients in our study who were assessed by abdominal ultrasound were also subjected to other imaging methods, and $52.2 \%$ of them were assessed and diagnosed only by abdominal USG.

In a study by Wertz et al [9] on 60 patients with $\mathrm{AC}$, abdominal ultrasound scanning was performed on 56 patients, abdominal tomography to 48 patients, and both imaging methods on 42 patients. In addition, 60 patients with no cholecystitis were evaluated with tomography and ultrasound as the control group. They found the sensitivity of tomography (85\%) superior to that of ultrasound $(68 \%)$ and noted no significant difference in the negative predictive value of both imaging methods. While tomography turned out to be positive in 10 patients out of 42 patients exposed to both tomography and ultrasound, no significant imaging was found on ultrasound for AC. In general, abdominal tomography is recognized as a more useful method for diagnosing $\mathrm{AC}$, but $65.2 \%$ of the patients diagnosed with cholecystitis in our study were scanned by ultrasound and $47.8 \%$ by abdominal tomography. Moreover, only $17.4 \%$ of the patients in our study were hospitalized after their abdominal tomography had been performed, while the patients assessed only by abdominal USG accounted for $52.2 \%$. It is well-documented that tomography is easier to perform, that the sensitivity of tomography is higher, that there is no contrast allergy in patients with suspected cholecystitis, and that tomography should be the primary imaging method in the absence of renal failure. However, ultrasound as an imaging method tends to be more widely used in elderly patients to avoid renal failure and the side effects of intravenous contrast media.

Cao et al [10] analyzed the complications of the patients with gallstones, reporting that $39.1 \%$ of the patients had diabetes, while $35.2 \%$ had hypertension, and that their cholesterol average was 188.32 $\pm 38.66 \mathrm{mg} / \mathrm{dl}$. As far as our study is concerned, the underlying comorbid diseases of the subjects were established as diabetes $(23.9 \%)$ and hypertension (6.5\%), and the mean blood cholesterol level was calculated as $141.14 \mathrm{mg} / \mathrm{dl}( \pm 31.33)$. In addition, while Goh et al [11] report that $12.2 \%$ of their patients diagnosed with $\mathrm{AC}$ were also afflicted with ischemic heart disease, this rate was found as $21.7 \%$ in our study. The presence of comorbid diseases reinforces the need for emergency surgery in patients admitted with abdominal pain complaints and diagnosed with cholecystitis, and a lower total cholesterol level may account for the low rate of acute calculous cholecystitis.

In our study, although the length of hospital stay did not differ significantly $(\mathrm{p}=0.286)$ between the within-Ramadan group with 5.6 days $( \pm 1.84)$ and the non-Ramadan group with 8.1 days $( \pm 5.6)$ possibly due to the small number of subjects, a clinical difference was nevertheless observed. We tend to attribute this situation to the fact that the total number of patients hospitalized in Ramadan was 10 .

In addition, the length of hospital stay for the patients in the intensive care unit amounted to 14.67 days $( \pm 4.51)$, while that of inpatients not hospitalized in the intensive care was 7.07 days $( \pm 4.84)$. The length of stay for three patients followed up in the intensive care unit was 19, 10, and 15 days, 
respectively. We assume that the lack of a significant difference between intensive care patients and inpatients was likely to result from the small number of patients in the intensive care unit and clinicalwise, the number of hospitalization days doubled. All three patients admitted within the Ramadan month were subjected to emergency operation. We also hold that hospitalization in the intensive care and advanced age of the patients may have contributed to the increase in the length of hospital stay.

\section{Limitations}

Admittedly, the generalizability of these findings is subject to certain limitations. An issue remaining unaddressed in this study is that approximately 36 years of data should be taken into consideration since Ramadan is routinely celebrated 10 days before the previous year. Another important limitation lies in the fact that only a specific region in Turkey was considered within the scope of our work, and there might inevitably be differences between the ways the Ramadan month is celebrated at different times and circumstances in other regions. Therefore, further research involving different regions should be undertaken in the future.

\section{Conclusions}

As a result, no significant difference was identified in terms of the frequency of $\mathrm{AC}$ in the Ramadan month in comparison to the Shaban and Shawwal months. Even though there was no significant difference regarding emergency surgery, length of hospital stay, and intensive care hospitalization, a clinical difference was nevertheless observed. We are of the opinion that the results might turn out to be statistically significant provided that the study is performed with a larger number of subjects.

\section{Conflicts of interest}

The authors have no relevant financial or non-financial interests to disclose.

\section{Ethics statement}

The approval for this study was granted from the non-interventional ethics committee of Pamukkale University with dated April 17, 2018, and numbered 2018/08.

\section{Funding}

No funds, grants, or other support was received.

\section{Author Contributions}

All of the authors declare that they all have participated in the design, execution, and analysis of the paper and approved the final version.

\section{References}

[1] Yokoe, M., Hata, J., Takada, T., et al. "Tokyo Guidelines 2018: diagnostic criteria and severity grading of acute cholecystitis (with videos)", J Hepatobiliary Pancreat Sci, 25(1), 41-54, 2018.

[2] Abbas, SM., Basalamah, AH. "Effects of Ramadhan fast on male fertility", Arch Androl, 16(2), 161-166, 1986.

[3] Hosseini, SV., Torabijahromi, M., Mosallaei, M., Sabet, B., Pourahmad, S. "The effect of season and Ramadan fasting on the onset of acute cholecystitis", Saudi Med J, 27(4), 503-506, 2006.

[4] Halpin, V. "Acute cholecystitis", BMJ Clin Evid, 08, 411, 2014.

[5] Kuroi, Y., Imazato, D., Yamazaki, K., Kasuya, H. "Acute cholecystitis in patients with stroke", Neurol India, 67(2), 439-441, 2019.

[6] Indar, AA., Beckingham, IJ. “Acute cholecystitis”, BMJ, 325(7365), 639-643, 2002. 
[7] Yu, H., Uyeda, JW. "Imaging of Acute Hepatobiliary Dysfunction”, Radiol Clin North Am, 58(1), 45-58, 2020.

[8] Abboud, PA., Malet, PF., Berlin, JA., Staroscik, R., Cabana, MD., Clarke, JR., Shea, JA., Schwartz, JS., Williams, SV. "Predictors of common bile duct stones prior to cholecystectomy: a meta-analysis", Gastrointest Endosc, 44(4), 450-455, 1996.

[9] Wertz, JR., Lopez, JM., Olson, D., Thompson, WM. "Comparing the Diagnostic Accuracy of Ultrasound and CT in Evaluating Acute Cholecystitis", AJR Am J Roentgenol, 211(2), 92-97, 2018.

[10] Cao, Z., Wei, J., Zhang, N., Liu, W., Hong, T., He, X., Qu, Q. "Risk factors of systematic biliary complications in patients with gallbladder stones", Ir J Med Sci, 189(3), 943-947, 2020.

[11] Goh, SNS., Chia, CLK., Ong, JW., Quek, JJX., Lim, WW., Tan, KY., Goo JTT. “Improved outcomes for index cholecystectomy for acute cholecystitis following a dedicated emergency surgery and trauma service (ESAT)", Eur J Trauma Emerg Surg, 2020. published online ahead of print, doi:10.1007/s00068-020-01308-1 